\title{
EMPRESARIAS Y AGENTES CULTURALES DEL SIGLO XIX. EL MODELO DE FAUSTINA SÁEZ DE MELGAR (1834-1895)
}

\author{
Virginia SEguí Collar \\ Universidad Nacional de Educación a Distancia
}

\begin{abstract}
En este artículo se aborda el estudio de la actividad cultural, editorial y empresarial realizada por la escritora Faustina Sáez de Melgar. Documentos inéditos de su archivo personal permiten un renovador acercamiento a su figura y a su labor profesional, desvelando su verdadera dimensión como mujer, escritora, periodista, empresaria y activista cultural que supera con creces su tradicional y reductora clasificación como escritora isabelina. La aclaración de relevantes datos personales y profesionales desvelan a una mujer emprendedora y con iniciativa propia que, paralelamente a su labor literaria, asumirá, a lo largo de toda su vida, novedosos retos en campos tradicionalmente masculinos.
\end{abstract}

PALABRAS ClaVE: Faustina Sáez de Melgar, editora, emprendedora, agente literaria.

Entrepreneurs and Cultural Agents from the 19th Century. Faustina Sáez de Melgar's Model

This article discusses the cultural, editorial and business activities carried out by the writer Faustina Sáez de Melgar. The study of as yet unpublished documents from her personal archive enables a renewed approach to her figure and her professional work, revealing her true dimension as a woman, writer, journalist, entrepreneur and cultural activist that far exceeds her traditional and reductionist classification as a typical mid-nineteenth-century woman writer. The clarification of important personal and professional data shows her as an independent enterprising woman who, in parallel to her literary work, assumed, throughout her life, challenges in traditionally masculine fields, new for a woman of her time.

KEY WORDS: Faustina Sáez de Melgar, publisher, entrepreneur, literary agent.

Una mirada renovadora sobre la figura de la escritora Faustina Sáez de Melgar (1834-1895) exige, en primer lugar, aclarar datos erróneos sobre la identidad de su marido y su posición social; datos que, al ser tenidos como ciertos, han depreciado su trayectoria profesional reduciéndola a la simple calificación de escritora isabelina (Sánchez-Llama, 2000: 28-29, 191). Sin que esta clasificación, en sí misma, pueda ser considerada peyorativa y aun reconociendo su adscripción literaria a la estética vigente en la época, lo cierto es que su verdadero perfil vital la aleja de este canon cuyo sentido reduccionista ha conducido a infravalorar su verdadera

Seguí Collar, Virginia (2019), "Empresarias y agentes culturales del siglo XIX. EI modelo de Faustina Sáez de Melgar (1834-1895)", Lectora, 25: 91-103. ISSN: 1136-5781 D.O.I.: 10.1344/Lectora2019.25.5, seguicollar@gmail.com

Recepció: 15 de desembre de 2018 - Acceptació: 15 d'abril de 2019 
dimensión como mujer emprendedora y activista cultural, desdibujando su faceta literaria y empresarial.

Sus deseos de convertirse en escritora fueron tempranos y contrarios a los de sus padres, lo que no hizo sino avivar sus intenciones, pese a la certeza de que cumplirlos exigiría sacrificio. Conoció a Valentín Melgar Chicharro en agosto de 1854, durante una visita a Guadalajara y, apenas un año después, el 16 de julio de 1855, contrajeron matrimonio (Seguí, 2016). Pilar Sinués (Sinués, 1860: 80) y J.E. Hartzenbuch, ${ }^{1}$ sus primeros biógrafos, mencionan este dato sin identificar claramente a su marido al no indicar el segundo apellido; omisión que ha motivado que se la vincule, sin ninguna base documental, con el aristócrata y acaudalado banquero Valentín Melgar Ceriola y que se atribuyan, a partir de ahí, los éxitos y el trabajo profesional de la escritora a la influencia y privilegiada posición de su marido. $^{2}$

Valentín Melgar Chicharro nació y vivió, hasta su matrimonio, en Guadalajara, en el seno de una familia de la clase media sin influencia social ni económica relevante. Tras su matrimonio, la pareja se instaló en Madrid con el convencimiento de que en la capital ambos encontrarían las mejores opciones profesionales (AFSM, C0066). Este matrimonio no fue del gusto de Silverio Rafael Sáez de Garillete, padre de Faustina, quien no acudió a la boda y probablemente dilató el pago de su dote agravando, con ello, la situación del matrimonio que solo contaba con los rendimientos que Valentín obtenía con la participación en alguna propiedad minera (AFSM, D0197), y quien no estabilizó su situación profesional hasta mediados de 1868, cuando entró en la Administración del Estado desempeñando destinos en la Península y, finalmente, en Ultramar hasta su jubilación en $1892 .{ }^{3}$

El error en la identidad del marido llevó a asociar a la escritora con posiciones políticas conservadoras y neocatólicas propias de la familia Ceriola (SánchezLlama, 2000: 192). La realidad, sin embargo, es otra, ya que Faustina y su familia apoyaron la causa liberal. Su padre era, en 1835, Subteniente de la Milicia Urbana en la Sección de Infantería, unidad que recobró pronto su nombre de Milicia Nacional. Su marido y sus hermanos pertenecieron al citado cuerpo defensor de la causa liberal e isabelina (AFSM, C0169). La primera Guerra Carlista (1833-1840) afectó directamente al pueblo natal de Faustina Sáez de Melgar que, en marzo de 1838 , sufrió ataques de guerrilleros carlistas que pusieron en grave peligro a la población (Cana, 2014). La familia vivió momentos de gran angustia que Faustina,

\footnotetext{
${ }^{1}$ Archivo de Faustina Sáez de Melgar, Sue0648. (Archivo de propiedad familiar. Se citará como AFSM).

${ }^{2}$ Véanse Simón Palmer (1993: 8), Sánchez-Llama (2000: 191; 2001: 137, 192, 300); Hibbs (2016: 348).

${ }^{3}$ Archivo General de la Administración. Leg. AGA 20782.
}

92 
pese a su corta edad, nunca olvidaría y que despertaron en ella un sentimiento de repulsa hacia los partidarios del pretendiente D. Carlos de Borbón, del que dejó constancia en algunas de sus obras (Sáez,1862; Sáez, 1872).

Su andadura literaria se inspiró en el camino seguido por las escritoras de la generación anterior que ya contaban con el reconocimiento público, como Carolina Coronado, Josefa Massanés, Amalia Fenollosa, etc., las cuales habían iniciado su actividad publicando poemas en periódicos y revistas. Sus biógrafos mencionan que su primer poema, "La paloma torcaz", apareció a finales de 1851 en El Correo de la Moda (Sinués, 1860: 79; Hartzenbusch, 1866: 9), provocando un gran revuelo familiar y el aumento, si cabe, de la oposición de sus padres hacia sus inclinaciones literarias. Ella perseveró en su labor y siguió enviando poemas a la prensa firmados, ahora, bajo seudónimos y/o anagramas. Su matrimonio le permitió escribir libremente e incrementar su actividad hasta conseguir un corpus significativo de poemas que, llegado el momento, le permitiría publicar su primer poemario, además de ampliar a otros géneros su actividad en busca del éxito literario y económico.

Su profesionalización como escritora coincidió con el desarrollo y modernización del sector editorial que trataba de superar estructuras gremiales heredadas y de adecuarse al mercado y a las leyes de la oferta y la demanda. La confusión entre los oficios de librero, impresor y editor era aún una constante en el panorama cultural español (Martínez Martín, 2003: 601). La escritora, consciente de las dificultades que cualquier escritor - y más cualquier escritora - tenía para publicar buscó soluciones alternativas para editar y difundir sus obras.

El estudio de su método de trabajo demuestra que Sáez de Melgar realizó un análisis detallado del sistema empleado por los profesionales del ramo para distribuir y rentabilizar sus publicaciones. Desde su primera obra, La Lira del Tajo (Sáez, 1859; AFSM, TP0010), y a lo largo de toda su vida, aplicó sistemáticamente una serie de acciones encaminadas a difundir, vender y controlar el beneficio de su obra, ya fuera esta literaria o periodística. Sus encargos al impresor eran siempre de un número significativo de ejemplares con sus correspondientes portadas, contraportadas, etc. y el prospecto informativo de la obra. El encuadernado inicialmente lo aplicaba únicamente al 50\% de los ejemplares impresos y el resto lo reservaba para adecuarlo a la demanda y/o para utilizarlo como regalo a los suscriptores de sus revistas, como sistema de fidelización (AFSM, F0040).

La necesidad de aumentar su visibilidad en el mundo literario y su búsqueda de nuevas formas de publicación, menos explícitas que la autoedición, la llevaron a fundar en Madrid, a finales de 1862, sin ayuda financiera externa, su primera empresa periodística: La Violeta. Revista Hispano Americana (1862-1867), con el apoyo expreso y material de su marido, todavía a la búsqueda de un empleo fijo, que además de aparecer como propietario y editor asumió tareas administrativas 
y contables. Pese a no aparecer como tal, Faustina añadió así a su faceta de editora de libros la de editora de revistas (Alonso, 2003: 591).

Sáez de Melgar presentaba sus publicaciones y actividades con un prospecto en el que informaba de su contenido, su temática y los puntos de adquisición y suscripción, en los que siempre se anunciaba la venta directa en su domicilio particular (AFSM, F0040). Sistemáticamente remitía un prospecto y un ejemplar de la publicación a librerías, redacciones de periódicos y revistas de mayor prestigio de la capital y provincias con el fin de asegurarse su difusión. Sus obras eran agrupadas bajo el formato de Bibliotecas o Colecciones con objeto de dotar a su dispar producción de una cierta unidad y con la intención de despertar en los lectores el deseo de poseer la serie completa de libros. Hacia 1860 creó La Biblioteca de Señoras, que mantuvo activa toda la década (AFSM, Sue0908). A mediados de 1878 la revitalizó en una segunda época en la que comercializó una nueva serie de obras (AFSM, Sue0189) que incluiría también en la colección Lecturas para las Damas. Biblioteca de Novelas Originales de Faustina Sáez de Melgar, todo un proceso de canonización personal en el que se sigue similar sistema de difusión y venta (AFSM, Sue0136). En 1880 individualizó sus publicaciones educativas en $\mathrm{La} \mathrm{Bi}$ blioteca Infantil (AFSM, Sue0983). Al igual que cualquier editor profesional, la autora ofrecía a libreros, impresores, editores o agentes comisionistas, con los que se relacionaba en España, Francia o Ultramar, corresponsalías y comisiones según el pedido de ejemplares. Con el fin de embellecer sus publicaciones y convertirlas en objetos artísticos además de textuales se interesó por las innovaciones del sector editorial. Para el lanzamiento de esta segunda fase de su Biblioteca de Señoras, en junio de 1878, invirtió cerca de 8.000 reales para modernizar las portadas de sus libros y hacerlos más atractivos. En palabras del impresor, Fernando de Rojas, las portadas al cromo con las que la escritora deseaba editar sus obras, si bien eran una novedad, encarecían la edición:

Efectivamente para una biblioteca de señoras es una novedad, pero creo que el precio es un poquito subido [...]. Hay que añadir á esto la encuadernación que tiene que ser igual al libro porque de otro modo no se puede charolar, y por la encuadernación quiere el librero Cid a $60 \mathrm{rs.} \mathrm{el}$ ciento; los tomos vendrán a costarle a V. de 1 r 3/4 á 2 rs. (AFSM, D0788; ortografía original)

Durante la publicación de La Violeta, Sáez de Melgar creó una colección especial asociada a la revista que utilizó para publicar obras suyas y de sus colaboradores como como regalo a los suscriptores o para su venta a través de la administración.

94

Lectora, 25 (2019): 91-103. ISSN: 1136-5781 D.O.I.: 10.1344/Lectora2019.25.5 
Faustina Sáez de Melgar iba adaptando su labor editora a sus sucesivas actividades, ya fueran estas literarias, periodísticas o educativas. Entre 1869 y 1871, años de funcionamiento del Ateneo Artístico y Literario de Señoras, ${ }^{4}$ reeditó La Violeta convirtiéndola en el órgano de difusión de la institución. Asumió la dirección de la revista y convirtió a los miembros de la sección literaria del Ateneo en sus redactores. Su proyecto era ambicioso pues su intención era editarla e imprimirla. Con el fin de valorar esta opción solicitó presupuesto a José Álvarez, profesional de las artes gráficas, quien, en febrero de 1870, le informó de que los costes de enseres y material supondrían un gasto de 2.812 rs (AFSM, D0111).

El elevado importe del proyecto impidió su instalación en el Ateneo que, pese a algunas afirmaciones (Simón Palmer, 2002: 21), se financiaba con pequeñas donaciones, las aportaciones de los socios y las pocas matrículas de las alumnas de pago. Su implantación podría haber supuesto un ahorro en los gastos del Ateneo si, con el tiempo, se hubiesen impreso en ella otros elementos como libros, carnés y otros documentos de uso habitual en la institución. La escritora, emprendedora por naturaleza y dotada de una mente práctica, quizás tuviera en mente la idea de convertir la imprenta en una escuela-taller donde las alumnas que así lo desearan aprendieran el oficio y acabaran por asumir su funcionamiento. La precaria situación económica del centro condicionó su cierre al finalizar el curso de 1870-1871.

En junio de 1871 estaba ya al frente de un nuevo proyecto periodístico: La Mujer. Revista de Instrucción General para el Bello Sexo (1871); su idea era centrase en la educación de la mujer superando el modelo de crónicas de moda y sociedad. Su prospecto anunciaba proyectos editoriales al establecer la distribución de una colección de novelas originales que se inició, desde el primer número, con su obra María. El hogar sin fuego. Cada ejemplar iba acompañado de ocho páginas de texto, de forma que al finalizar las entregas y tras facilitar las portadas, contraportadas, índices, láminas, etc., el lector que lo deseara podía encuadernar la obra.

En enero de 1876, se dio de alta en la Asociación de Artistas y Escritores Españoles que iniciaba su andadura oficial, asignándosele el número 452 (Porpetta, 1986: 55; AFSM, D0178). Con ello pretendía, al igual que cualquiera de sus miembros independientemente de su sexo, reafirmar su estatus profesional y la protección que la Asociación ofrecía a sus individuos y familias.

La educación de la mujer fue siempre un tema clave que afectó transversalmente todas las actividades de la escritora; así lo indicaba en el discurso de

\footnotetext{
${ }^{4}$ Institución educativa fundada y dirigida por la escritora a finales de 1868, en la que se impartieron clases, se realizaron sesiones recreativas y conferencias destinadas a la educación de la mujer.
} 
presentación de su proyecto educativo, L'École Professionnelle des Jeunes FillesAsociación Santa $\mathrm{Fe}^{5}$ en 1886:

Pido y deseo y trabajo únicamente por la instrucción de la mujer, esa fue la aspiración de toda mi vida. Antes de fundarse aquí en Madrid, la Escuela de Institutrices, las Conferencias de la Universidad y otros centros destinados para la educación de la mujer, yo fui la primera en señalar esa necesidad; fundé el Ateneo de Señoras y tuvimos más de cuatrocientas alumnas, que aprendieron en nuestras clases de enseñanza superior y de Bellas Artes. (AFSM, D0379)

Declaraba así su mayor aspiración destacando la importancia que, a su juicio, tuvo la fundación del Ateneo Artístico y Literario de Señoras en 1868; presentándolo como ejemplo inspirador y antecedente inmediato de iniciativas realizadas, poco después, por personas con mayores posibilidades políticas, económicas y administrativas, en una clara referencia al krausista Fernando de Castro, rector de la Universidad Central de Madrid (Simón, 1982: 13-14; 2002: 20-22).

Con frecuencia se han considerado el pensamiento y la fundación del Ateneo de Señoras como una iniciativa krausista, relegando a la escritora a un segundo plano y designando a Fernando de Castro como inspirador y fundador (SánchezLlama, 2000: 41, 195); nada más alejado de la realidad, según se desprende de las palabras del religioso, ya rector de la Universidad Central, al aceptar pronunciar el discurso de apertura del Ateneo:

Señora $\mathrm{D}^{\mathrm{a}}$ Faustina Saez de Melgar. [...] He recibido y leído su muy atenta del 25, he visto el Arto que le acompaña, y quedo enterado de los Estatutos, aplaudo y celebro lo ultimo, mas no asi el contenido de aquella, $\mathrm{pq}^{\mathrm{e}}$. las ideas que v. manifiesta, pura bondad, respecto de mi persona, no corresponden á lo poco q. yo valgo, y menos para el caso de su Ateneo, siendo yo eclesiástico, y poco conocedor por tanto de la vida y educación de la mujer. [...] Respecto del Discurso inaugural me encuentro perplejo entre el deber de caballero y la dificultad de las razones incluidas y la mayor aun de $\mathrm{q}^{\mathrm{e}}$. no he [ilegible] siempre mostrarme en publico y ser objeto de censura o alabanza. Cuando llegue el momento de la invitación oficial tendré pensado y resuelto lo q. deba hacer. En tanto que v. como el Ateneo de Señoras q. con tal prontitud y celo ha sabido v. fundar pueden disponer

\footnotetext{
${ }^{5}$ Asociación fundada por la escritora con el apoyo de un grupo de damas hispanoamericanas con el fin de crear escuelas profesionales, en Madrid, París y Barcelona, para la formación de las huérfanas de los Empleados Civiles (AFSM, D0338, Sue0016).
}

96

Lectora, 25 (2019): 91-103. ISSN: 1136-5781 D.O.I.: 10.1344/Lectora2019.25.5 
de mi inutilidad. [...] Fernando de Castro. Hoy 28. (AFSM, D0123; ortografía original)

Sus palabras son claras y, sin duda, le desvinculan de la fundación del Ateneo haciendo, incluso, difícil entender sus posteriores iniciativas destinadas a la educación de la mujer sin que una inspiración ajena le hiciera comprender la necesidad y el posible éxito de una empresa de esta índole.

Faustina, sin recursos económicos propios, basó sus proyectos educativos en el espíritu asociativo ligado al pensamiento general del movimiento liberal (Burguera, 2012: 35) que, tras el triunfo de la Revolución de 1868 retomaría su antiguo esplendor; al igual que la educación considerada de vital importancia para la transformación de la sociedad (Andreu, 2017: 572), pensamiento compartido por la escritora, pese a su interés por desvincularse de la política partidista (Sáez, 1869: 23). Siempre fue plenamente consciente de que el éxito de su iniciativa educativa, asociativa y filantrópica fue un ejemplo y un prometedor camino a seguir para aquellos que al disponer de más influencia y posibilidades económicas tenían mayores oportunidades de éxito. En sintonía con la línea abierta por las denominadas "damas del liberalismo respetable” (Burguera, 2012), Sáez de Melgar siempre mostró gran interés y sensibilidad por los problemas de la sociedad en general y de las mujeres en particular, participando en iniciativas y proyectos encaminados a mejorarlos. Fue Consiliaria de la Asociación de las Escuelas Dominicales (AFSM, D0096), colaboró en la Asociación de Hospitales de Niños, proyecto promovido por la duquesa de Santoña, o como presidenta de la Junta Auxiliar del distrito Centro de Madrid (AFSM, D0336). Apoyó la creación de un canal de regadío en la vega del Tajo para favorecer el desarrollo agrícola de la zona y, ante las demoras en su construcción, envió artículos a la prensa madrileña (Sáez, 1868; 1878) y cartas al Ministro de Fomento urgiendo su realización y denunciando la actitud de las constructoras adjudicatarias que, con concesiones subsidiarias, desvirtuaban y dilataban el proyecto (AFSM, D0309).

Consecuente con sus inquietudes sociales, apoyó sin ambigüedades el movimiento abolicionista. En octubre de 1863, Julio L. Vizcarrondo, representante de la Sociedad Abolicionista Española, le trasladó el contenido de los manifiestos que señoras de sociedades abolicionistas de Londres, Birmingham, Edimburgo, Newcastle y París le habían entregado para invitar a las madrileñas a unirse a su lucha. Faustina, pese a los perjuicios que su posicionamiento en este tema pudieran acarrearle, se unió al movimiento y los publicó en La Violeta; acudió a las reuniones abolicionistas y participó, como vocal, en la Junta de la Sociedad de Señoras Protectoras de los Esclavos (AFSM, D0133, D0811). En diciembre de 1865 fue la encargada de animar a las señoras a unirse al movimiento y a acudir a la reunión 
abolicionista que se celebraría el día 10 en el teatro Variedades de Madrid (AFSM, Sue0273).

El interés por la educación de la mujer y por la edición llevó a Sáez de Melgar a formar parte de la Sociedad de Escritores Protectores de las Letras, fundada en 1878 por la duquesa de Carlet (AFSM, Sue0383) con el fin de proteger la labor intelectual y fomentar la lectura. Faustina, como secretaria de la Junta de Damas que se encargaba de la Sección Segunda, dedicada al Fomento de las Buenas Lecturas, colaboró en su gestión cediendo, incluso, dos habitaciones en su domicilio particular para la instalación provisional de la biblioteca destinada a promover el préstamo domiciliario (AFSM, D0253). La sociedad exponía sus objetivos "de protección al trabajo intelectual y creación de bibliotecas de lectura gratuita a domicilio" y solicitaba apoyo económico y/o material mediante un oficio-circular que remitía a escritores, artistas y demás miembros activos de la sociedad para obtener fondos para la realización de sus fines. La labor editorial cumplía dos objetivos: proteger el trabajo intelectual y fomentar la lectura dotando de libros a las bibliotecas. El archivo de la escritora conserva documentos en los que aparecen gastos de encuadernación, imprenta, litografía, etc. y una relación de obras impresas (AFSM, D0760, D0761).

En septiembre de $1880,{ }^{6}$ considerando la negativa de su marido a que la familia se trasladara con él a sus destinos ultramarinos, Sáez de Melgar se instaló en París junto a sus dos hijas con el fin de ampliar sus horizontes profesionales y perfeccionar la educación de estas. En octubre le llegó la oferta del editor barcelonés Juan Pons de dirigir y coordinar la publicación Las mujeres españolas, americanas y lusitanas, pintadas por sí mismas (Sáez, 1882a), propuesta que aceptó pese a las dudas que le suscitaban la premura y las condiciones del empresario, así como su indefinición en el tema económico; el prestigio que podía adquirir al convertirse en la directora literaria de la obra con mayor participación femenina publicada hasta entonces motivó su aceptación. Sus sugerencias al editor mejoraron la publicación ampliando el ámbito geográfico de los tipos al incluir a las lusitanas, las biografías de las participantes y la entrega de sus retratos de manera que la realidad de sus vidas y actividades sirviera de contrapunto a unos artículos costumbristas limitados por el editor al querer dar a su obra un carácter "sumamente moral y hasta de doctrina en lo posible” (Seguí, 2002: 230-232, 235).

La importancia que para su desarrollo profesional significaba darse a conocer y adquirir un cierto estatus en el mundo literario francés hizo que uno de sus primeros pasos en la capital francesa fuera contactar con la Association Littéraire et Artistique Internationale; fundada en junio de 1878 (Pouillet, 1889: 1), encargada

\footnotetext{
${ }^{6}$ El 5 de septiembre de 1880 la revista París-Conférence, en su sección "Nouvelles Littéraires", daba cuenta de su llegada a París.
}

98

Lectora, 25 (2019): 91-103. ISSN: 1136-5781 D.O.I.: 10.1344/Lectora2019.25.5 
de regular y proteger la propiedad artística y literaria dando cabida a profesionales de todas las nacionalidades (Lermina, 1889: xiv-xix). Así, se aseguraba una relación fluida con sus compañeros de Letras y, a la vez, una información puntual y de primera mano de las novedades legales que podían afectar a sus actividades en Francia.

Sus primeros objetivos periodísticos se centraron en la fundación o desempeño de la dirección literaria de revistas en castellano destinadas al público de habla hispana con residencia en Europa y/o Ultramar. En enero de 1881 se asoció con la firma Silvestre Rouveirolles \& Cía (AFSM, CT0011), propietaria de los Almacenes Saint-Joseph y editores de la revista Paris Charmant, quienes deseaban publicar una versión de su revista en lengua hispana; Sáez de Melgar se convertía así en su directora literaria. Comenzó a publicarse en febrero de 1881 y a partir de 1882 pasó a denominarse París Charmant Artistique (AFSM, Sue0129).

En diciembre de 1881 la escritora fundó La Canastilla Infantil, en la que aparece como propietaria y directora. En enero de 1883 cedió el $50 \%$ de la empresa a José Y. Ferrer, que se convirtió en el administrador mientras ella continuaba su labor como directora literaria. La publicación pasó a denominarse La Canastilla del Hogar (AFSM, CT003).

Al no cumplir sus expectativas con estas revistas, Sáez de Melgar decidió, en 1884, refundar La Violeta. Gaceta Ilustrada para las Madres de Familia (AFSM, Sue0131) en su fase parisina; en esta segunda época, imprimió 4.000 prospectos para su difusión y tiradas de 1.000 ejemplares de cada número (AFSM, Sue0839). Su fase editora volvió a tomar forma al incentivar las suscripciones con el regalo de sus novelas originales. Para conocer el importe de la edición de 5.000 ejemplares de alguna de ellas solicitó presupuesto a la firma Paul Dupont, Société Anonyme d'Imprimerie et Libraire, encargada de imprimir la revista, que el 17 de enero de 1884 estimaba el coste en 1.786 francos con una disminución de 300 fr. limitando a 3.000 los ejemplares encuadernados (AFSM, D0817), lo que da una idea de sus expectativas de venta. En sus comunicaciones con libreros y agentes utilizaba oficios con membrete en los que aparecía la relación de sus obras incluidas ahora en La Biblioteca de las Madres de Familia, vinculándola así con su revista (AFSM, F0184).

Esta labor no le impidió continuar con sus colaboraciones habituales en la prensa española, básicamente madrileña, con la que seguía manteniendo contacto y en la que publicaba poesías, artículos o folletines, iniciando, al tiempo, lo que parece una actividad de corresponsal en el extranjero al publicarse sus artículos sobre moda y sociedad como Crónicas Parisinas firmadas con su nombre y/o con alguno de sus pseudónimos: Artemisa (Sáez, 1882b), Baronesa de Villmont o Willmont (AFSM, D0122) y/o La Parisiense (Sáez, 1886). 
Su mayor reto profesional llegó con su integración, como Directrice de la Sección Española, en el Centre Littéraire Internationale, empresa vinculada al escritor y periodista francés Alfred Sirven, fundador de Le Correspondant Français, dedicada a la compra y venta de originales, su ilustración y traducción. Desde este cargo acometió su trabajo como agente literaria para algunas editoriales españolas que buscaban en el país vecino novelas originales de éxito con las que nutrir sus colecciones. Esta actividad, a veces, incluía la traducción de la obra y/o la compraventa de grabados originales destinados a su ilustración y la negociación, con librerías y corresponsales, para la venta y depósito de publicaciones (AFSM, D0028).

En 1862 había iniciado su labor traductora vinculada a la fundación de La Violeta, un complemento necesario para dar calidad a la revista y para su economía empresarial (Sánchez-Llama, 2000: 166). Traducía obras de pequeña entidad, relatos cortos, leyendas, etc. tomados de revistas francesas a las que estaba suscrita ${ }^{7}$ que calificaba como arreglos o traducciones libres. Su colaboración con La Moda Elegante. Periódico de las Familias (1861-1923), en la década de 1860 (AFSM, D0847), incrementó este trabajo dado el gusto de su propietario, Abelardo de Carlos, por las traducciones. Esto supuso un incremento en el número de traducciones que, además, adquirieron mayor complejidad y extensión. Entre ellas podemos citar la traducción de Los vecinos de Darlingen, novela del belga Hendrik Conscience (Sáez, 1867a; AFSM, D0575) y las de los relatos Las gavillas de la reina Blanca (Sáez, 1866a), original de A. Jadin (AFSM, Sue0730), La muda (Sáez, 1866b) de Henri Vierne (Vierne, 1862), La Virgen de las rosas (Sáez, 1867) del Vicomte Oscar Philippe François Joseph de Poli (Poli, 1863), entre otras. Este trabajo presupone su conocimiento del francés, idioma básico desde el que traducía. No obstante, Faustina inició el aprendizaje del alemán unos meses antes de trasladarse a París, con la intención de poder realizar, en un futuro próximo, traducciones directas de este idioma sin tener que recurrir a la mediación del francés (Fastenrath, 1880: 9).

Como agente literaria mantuvo contactos con escritores franceses, los más famosos del momento, negociando con ellos la adquisición de los derechos de traducción al castellano, y a veces al portugués, de sus obras. Destaca su trabajo para la editorial Rivadeneira, propiedad de Abelardo de Carlos y dirigida por su hijo Alfredo, con quien mantuvo correspondencia sobre las novedades de mayor éxito en Francia y las obras y los autores que le interesaban para su Biblioteca de Autores Contemporáneos (AFSM, D0053-D0061; D0078; D0080; D0492-D0497; D0558).

Sirven, Levérdier, Lermina, Zola, Niboyet, Belot y Berthet son algunos de los escritores con los que mantuvo contacto para la adquisición de derechos de

\footnotetext{
${ }^{7}$ La biblioteca de Sáez de Melgar contiene varios tomos de algunas revistas como Journal des Demoiselles, Le Conseiller des Dames y des Demoiselles, etc.
} 
traducción. Su trabajo más significativo fue la compra a Zola, a finales de 1882, de los derechos de $A u$ bonheur des dames (AFSM, D0714), poco después de que apareciese publicada en la célebre revista francesa Gil Blas, si bien el autor exigió el compromiso de que la versión española no fuera publicada hasta que la editorial Charpentier no la editara en Francia. En marzo de 1883 vendió los derechos de traducción a Alfredo de Carlos (AFSM, D0278) quien publicó la obra en junio de 1885 bajo el título $A$ la dicha de las damas (Sin firma, 1885). El Cosmos Editorial publicó su traducción de la obra de Pierre Zaccone Les drames de la bourse (Zaccone, 1884). Editoriales francesas también le encargaron la traducción de obras que les interesaba publicar en español; entre ellas Le mond, et ses usages de Mme. de Waddeville para el sello A. Hennuyer y Les voisins de Federica Bremer para Garnier Frères.

Faustina Sáez de Melgar regresó definitivamente a España en 1887, donde retomó sus actividades habituales y su trabajo de traductora, del que son una muestra representativa algunos cuentos de Carmen Silva, seudónimo de la reina de $\mathrm{Ru}$ manía, publicados por el sello El Cosmos Editorial bajo el título Flores y perlas (Sáez, 1889). El proyecto incluía un segundo tomo que, finalmente, no vio la luz por lo que la escritora optó por darlo a la prensa como folletín en El Día, bajo el título Dibujos a pluma (Sáez, 1892).

Este trabajo no es más que un simple esbozo de algunas de las actividades de Faustina Sáez de Melgar que nos permiten descubrir en ella una mujer activa y emprendedora en todos los ámbitos de la cultura y la sociedad. Su trayectoria vital y profesional desmiente la visión que hasta ahora se ha tenido de la autora pues, al basarse más en sus textos que en el conjunto de sus actividades, sólo confirmadas a la luz de la documentación de su archivo personal, se ha desvirtuado el valor y las dimensiones de su emprendimiento cultural en el último tercio del siglo XIX.

\section{ARCHIVOS CONSULTADOS}

AFSM: Archivo Privado Faustina Sáez de Melgar. Propiedad familiar.

Archivo Municipal Villamanrique de Tajo.

AGA: Archivo General de la Administración

\section{REFERENCIAS BIBLIOGRÁFICAS}

Alonso, Cecilio (2003), "La prensa y el libro", Historia de la edición y de la lectura en España, 1473-1914, Víctor Infantes de Miguel, François López y JeanFrançois Botrel (dirs.), Madrid, Fundación Germán Sánchez Ruipérez: 591600 . 
Andreu, Xavier (2017), "La cultura”, Historia contemporánea de España, vol. I, Jordi Canal (dir.), Madrid, Taurus: 509-578.

Burguera, Mónica (2012), Las damas del liberalismo respetable, Madrid, Cátedra.

Cana, Fernando (2014), "Una noche funesta", Fernando Cana. Blog de Historia, 28/08/2018. <//fernandocana.es/una-noche-nefasta/>

Fastenrath, Juan (1880), "El poeta y crítico Rodolfo Gottschall”, La América, 5: 910.

Hartzenbusch, Juan E. (1866), “Doña Faustina Sáez de Melgar”, La Violeta, 199: 296-299.

Hibbs, Solange (2016), "Faustina Sáez de Melgar (1864-1895): Una escritora y traductora fronteriza entre sombras y luces", Autores traductores en la España del siglo XIX, Francisco Lafarga y Luis Pegenaute (eds.), Madrid, Kassel: 345358.

Lermina, Jules (1889), "Composition du Bureau”, Association Littéraire \& Artistique Internationale, París, Bibliothèque Chacornat: xiv-xix.

Martínez Martín, Jesús A. (2003), “Editores y empresas editoriales”, Historia de la edición y de la lectura en España, 1472-1914, Víctor Infantes de Miguel, François López y Jean-François Botrel (dirs.), Madrid, Fundación Germán Sánchez Ruipérez: 301-303.

Porpetta, Antonio (1986), Escritores y artistas españoles, Madrid, Asociación de Escritores y Artistas Españoles.

Pouillet, Eugène M. (1889), "Résumé", Association Littéraire et Artistique Internationale, París, Bibliothèque Chacornat: 1-45.

Sáez de Melgar, Faustina (1859), La lira del Tajo, Madrid, Imprenta de Bernabé Fernández.

-(1862), Matilde o el Ángel de Valde Real, Madrid, Imprenta de Manuel de Rojas.

—(1866a) (trad.), “Las gavillas de la reina Blanca”, La Moda Elegante, Cádiz, 22: 171-172.

_(1866b) (trad.), "La muda”, La Moda Elegante, Cádiz, 36: 284-286. [AFSM, D0123]

-(1867a) (trad.), “Los vecinos de Darlingen”, La Moda Elegante, Cádiz, 31: 247248. [AFSM, D0123]

—(1867b) (trad.), "La Virgen de las rosas”, La Moda Elegante, Cádiz, 47: 375-376; 48: 382-383; 49: 391; 50: 398-399. [AFSM, D0123]

-(1868), "Canal del Tajo", La Nueva Iberia, 1 (206).

-(1869), Memoria del Ateneo de Señoras leída en Junta General celebrada el día 27 de junio de 1869 por la presidenta y fundadora, Madrid, Imprenta de los Sres. de Rojas.

102

Lectora, 25 (2019): 91-103. ISSN: 1136-5781 D.O.I.: 10.1344/Lectora2019.25.5 
-(1872), Rosa la cigarrera de Madrid, Barcelona, Juan Pons.

-(1875), María. El hogar sin fuego, Madrid, La Iberia.

-(1878), "Villamanrique de Tajo y su canal", El Popular, 2231.

-(1882a) (dir.), Las mujeres españolas, americanas y lusitanas, pintadas por sí mismas, Barcelona, Juan Pons.

-(1882b), "Crónica de París”, El Correo de la Moda, 20: 154-155.

-(1886), "Salones y Modas de París”, El Día, 2888: 2.

Sánchez-Llama, Íñigo (2000), Galería de escritoras isabelinas. La prensa periódica entre 1833 y 1895, Madrid, Cátedra.

-(2001), Antología de la prensa periódica isabelina escrita por mujeres (18431894), Universidad de Cádiz.

Seguí, Virginia (2001), "Una mirada sobre sí mismas”, Luchas de género en la Historia a través de la imagen. Tomo III, Teresa Sauret y Amparo Quiles (eds.), Málaga, Diputación Provincial de Málaga: 227-250.

-(2017), "Faustina Sáez de Melgar (Villamanrique de Tajo, Madrid, 1834 Madrid, 1895) [Semblanza]", portal Editores y Editoriales Iberoamericanos (siglos XIX-XXI), EDI-RED, Alicante, Biblioteca Virtual Miguel de Cervantes. $<$ http://www.cervantesvirtual.com/obra/faustina-saez-de-melgar-villamanriq ue-de-tajo-madrid-1834--madrid-1895-semblanza-776628/>

Simón Palmer, María del Carmen (1982), La mujer madrileña del siglo XIX, Madrid, Artes Gráficas Municipales: 13-14.

-(1993), Revistas femeninas madrileñas, Madrid, Artes Gráficas Municipales: 2022.

-(2002), Actividades públicas de las madrileñas en la I República, Madrid, Artes Gráficas Municipales.

Sin firma (1885), "Libros presentados a esta redacción por autores y editores", La Ilustración Española y Americana, Año XXIX, 1: 14.

Sinués de Marco, María del Pilar (1860), "Escritoras españolas. Faustina Sáez de Melgar”, El Bello Ideal: 79-80.

Vierne, Henri (1862), “La Muette”, Le Conseiller des Dames et des Demoiselles, XVI. <http://www.bmlisieux.com/archives/lamuette.htm>

Zaccone, Pierre (1884), Los dramas de la bolsa, Madrid, El Cosmos Editorial. 